\title{
Sports Skill Analysis using Motion Frequency
}

\author{
Toshiyuki MAEDA ${ }^{1,2}$, Takeshi MATSUDA ${ }^{3}$, and Masumi YAJIMA ${ }^{4,2}$ \\ ${ }^{1}$ Department of Management Information, Hannan University, Japan \\ ${ }^{2}$ School of Computing Science, Newcastle University, United Kingdom \\ ${ }^{3}$ Department of Information Security, University of Nagasaki, Japan \\ ${ }^{4}$ Department of Economics, Meikai University, Japan
}

\begin{abstract}
This paper addresses sports skill discrimination using motion picture data, focused on volleyball attack skill. We attempt to certify the hypothesis that expert skills have relatively low frequency motions rather than novice skills as the similarity of human postural control. For this purpose we proceed experiments and analyze sports skills as for frequency of motion using time series motion pictures of volleyball attacks. In this paper, volleyball play is analyzed with motion picture data recorded by hi-speed cam-coder, where we do not use physical information such as body skeleton model, and so on. Time series data are obtained from the motion picture data with four marking points, and analyzed using Fast Fourier Transform (FFT) and clustering data mining method. As the experiment results, we have found that y-axes of novice data may have more highfrequency data, and that implies novice motions may have high frequency motions, and that may support our hypothesis.
\end{abstract}

Keyword: Time Series Data, Sports Skill Analysis, Motion Picture, Fast Fourier Transform

\section{INTRODUCTION}

Many researchers use models of body or skeleton structure with physical information for activity or biomechatronical data skill researches, such as [1], and so on.

Matsumoto et al. describe, for example, that skilled workers skills which have actually internal models of structured skill architecture and they choose an action process from internal models adjusted with environment [2]. It is even though hard for skilled workers to represent internal models by themselves. They reflect involuntarily their own represented actions, and achieve highly technical skills with internal models.

So far, we had studied table tennis skill, focused on forehand strokes, which exemplifies sports action, and classify skill models using motion picture data analysis without body nor skeleton structure model. We had evaluated those into three play levels as expert/intermediate/novice, and classify the models using data mining technologies [3], [4]. We furthermore had an attempt to apply our research framework to other sports skills, such a personal sports skill identification using time series motion picture data, focused on volleyball [5].

There are, on the contrary, some researches for human postures. For instance, Nomura et al. [6] present that ground reaction force during human quiet stance is modulated synchronously with the cardiac cycle through hemodynamics. This almost periodic hemodynamic force induces a small disturbance torque to the ankle joint, which is considered as a source of endogenous perturbation that induces postural sway. Here they consider postural sway dynamics of an inverted pendulum model with an intermittent control strategy, in comparison with the traditional continuous-time feedback controller. They examine whether each control model can exhibit human-like postural sway, characterized by its power law behavior at the low frequency band $0.10 .7 \mathrm{~Hz}$, when it is weakly perturbed by periodic and/or random forcing mimicking the hemodynamic perturbation. They show that the continuous control model with typical feedback gain parameters hardly exhibits the human-like sway pattern, in contrast with the intermittent control model. Further analyses suggest that deterministic, including chaotic, slow oscillations that characterize the intermittent control strategy, together with the small hemodynamic perturbation, could be a possible mechanism for generating the postural sway.

This implies similar postural control may exist in sports skills as well as usual motion control, and that means expert skills have lower frequency motion comparing to novice one. We investigate the above hypothesis with sports skill analysis of motion frequency using time series picture data.

\section{RELATED WORKS}

Wilkinson [7] describes that qualitative skill analysis is an essential analytic tool for physical educators and refers to a process in which a teacher identifies discrepancies between the actual response observed and the desired response. Providing instruction for preserving teachers regarding how to recognize errors has been largely neglected in teacher preparation. The purpose of this study was to evaluate an alternative approach for teaching qualitative skill analysis to undergraduates. The study evaluated the effectiveness of a visual-discrimination training program. The subjects were 18 undergraduate students. The visual-discrimination training program was introduced using a multiple-baseline design across three volleyball skills: the forearm pass, the overhead pass, and the overhead serve. After the introduction of each instructional component, subjects made abrupt improvements in correctly analyzing the volleyball skill. This approach for teaching qualitative skill analysis is one alternative to the conventional techniques currently being used in professional preparation. 
Watanabe et al. [8] show a method for the measurement of sports form. The data obtained can be used for quantitative sports-skill evaluation. Here, they focus on the golfdriverswing form, which is difficult to measure and also difficult to improve. The measurement method presented was derived by kinematic human-body model analysis. The system was developed using three-dimensional (3-D) rate gyro sensors set of positions on the body that express the 3-D rotations and translations during the golf swing. The system accurately measures the golf-driver-swing form of golfers. Data obtained by this system can be related quantitatively to skill criteria as expressed in respected golf lesson textbooks. Quantitative data for criteria geared toward a novice golfer and a mid-level player are equally useful.

Barzouka et al. [9] examine the effect of feedback with simultaneous skilled model observation and self-modeling on volleyball skill acquisition. 53 pupils 12 to 15 years old formed two experimental groups and one control group who followed an intervention program with 12 practice sessions for acquisition and retention of how to receive a ball. Groups received different types of feedback before and in the middle of each practice session. Reception performance outcome (score) and technique in every group were assessed before and at the end of the intervention program and during the retention phase. A 3 (Group) $\times 3$ (Measurement Period) multivariate analysis of variance with repeated measures was applied to investigate differences. Results showed equivalent improvement in all three groups at the end of the intervention program. In conclusion, types of augmented feedback from the physical education teacher are effective in acquisition and retention of the skill for reception in volleyball.

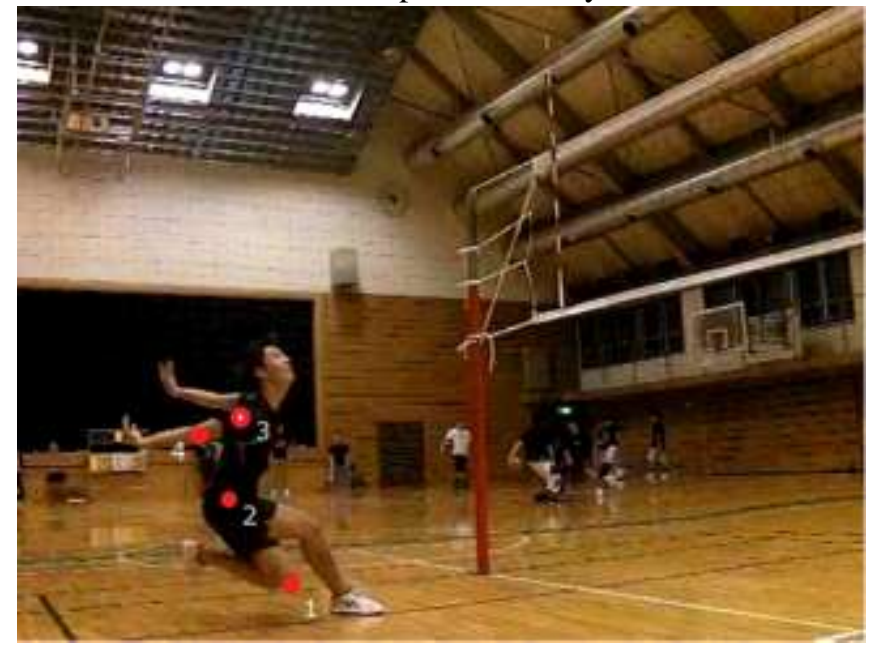

FIGURE 1. Measurement markings.

\section{EXPERIMENT}

We here make a target on volleyball attacks, and analyze volleyball skills from observed motion picture data and skill evaluation with represented actions.
For this research, we have recorded motion pictures of six subjects who are university students. As skill evaluation of representing action, we classify two levels as follows;

- Expert class: members of volleyball club at university,

- Novice class: inexperienced students.

In this experiment, the subjects are three expert and three novice-level ones. Figure 1 shows positions of marking setting.

Each player is marked at four points on the right arm as;

1) Left knee,

2) Right waist,

3) Right shoulder, and 4) Right elbow.

We have recorded swing traces of stacks using a high-speed cam-corder (resolution: $512 \times 384$ pixel and frame-rate: 300 frames per seconds) installed besides of the players. In playing in 5 minutes, several attack motions are recorded for each player.

\section{SKILl ANALYSIS}

In the recorded motion pictures, 210 to 360 frames are retrieved from the beginning of take-back to the ball until the end of the attack. We have then distributed two dimensional axes positions (pixel values) of four marking points for each frame, where the starting point is set at the shoulder position of the first frame. We analyze time series data of each axis using fast Fourier transform (FFT).

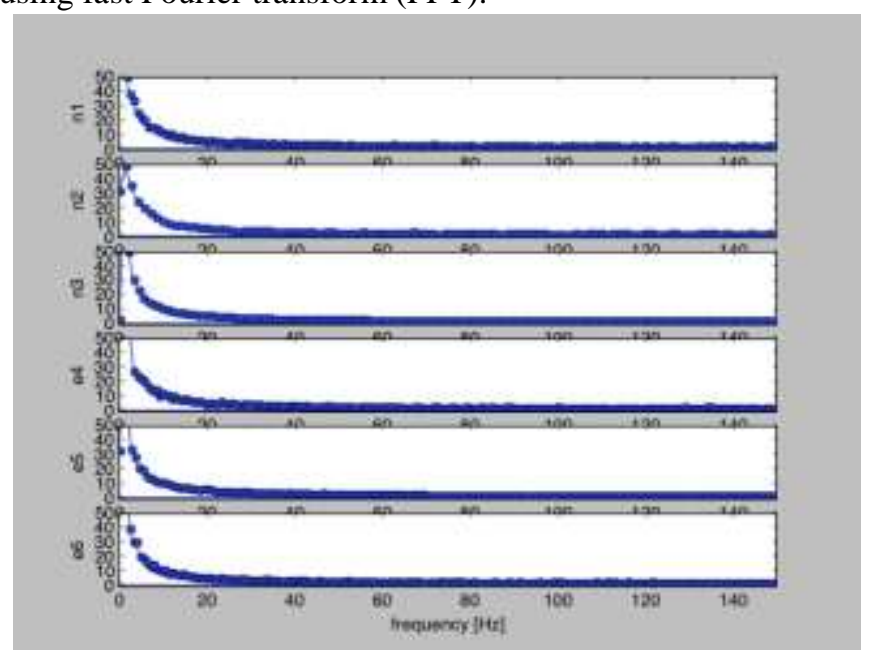

FIGURE 2. Frequency analysis for left-knee $\mathrm{X}$-axis of 6 subjects.

Figure 2 shows frequency spectrums of all six subjects for left-knee X-axis. In this figure, $\mathrm{n} 1, \mathrm{n} 2, \mathrm{n} 3$ are from the data of novice subjects, and e4,e5,e6 are of expert subjects. This figure may roughly suggest novice data tend to have high frequency. 
For further analysis, we focus on one novice (n2) and one expert (e4) subjects and present some spectrums of each axis as below, Figure 3, 4, respectively.

In those figures, especially figures of 3 novice data of leftknee $\mathrm{y}$-axes have more high-frequency data. That implies novice motions may have high frequency motions.

Furthermore, we make an attempt to classify frequency data using hierarchical clustering technique, and some have fairly

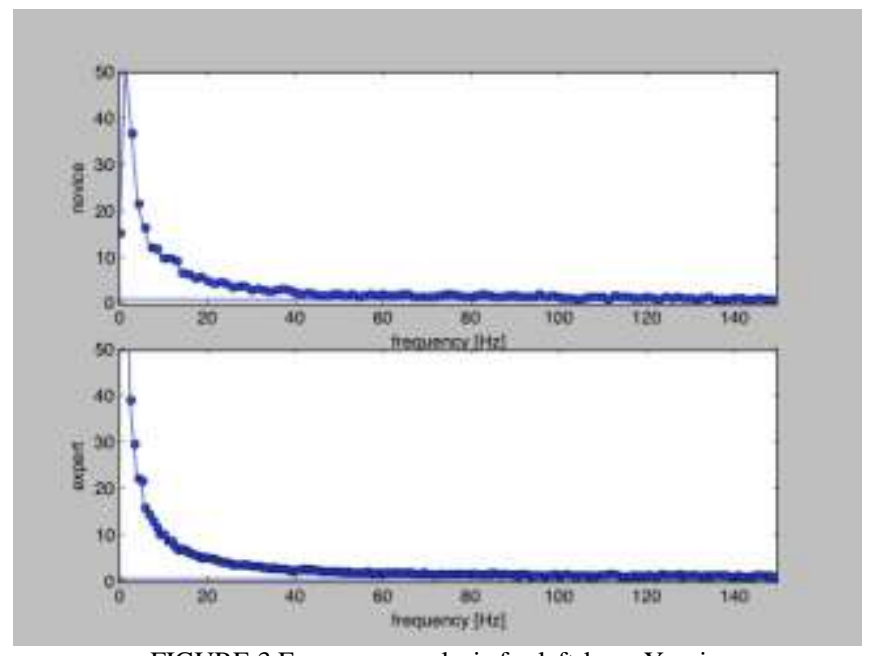

FIGURE 3.Frequency analysis for left-knee Y-axis.

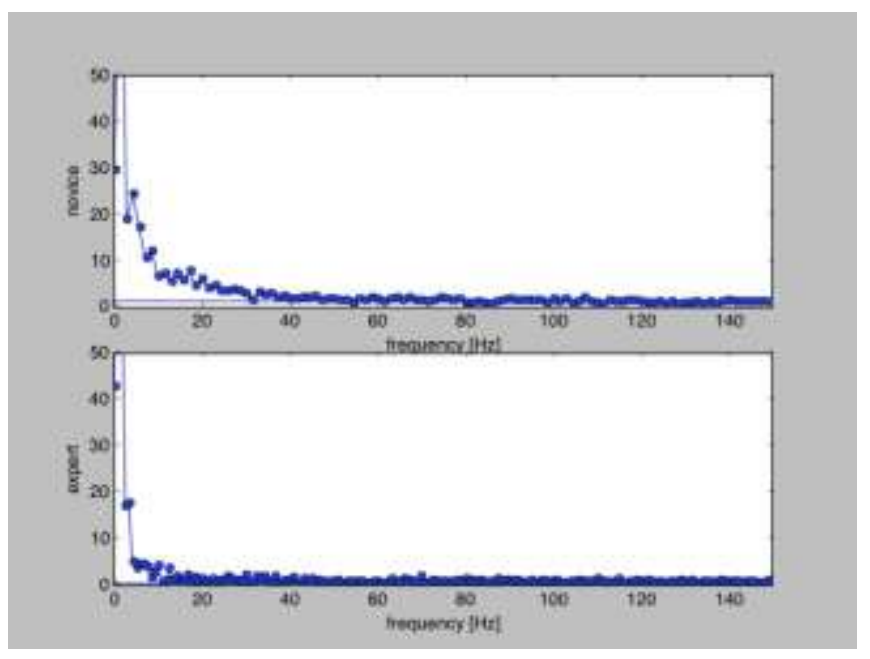

FIGURE 4. Frequency analysis for right-shoulder X-axis.

good results. For instance, Figure 5 shows the result of left knee Y-axis, and this implies novices $(\mathrm{n} 1, \mathrm{n} 2, \mathrm{n} 3)$ and experts $(\mathrm{e} 4, \mathrm{e} 5, \mathrm{e} 6)$ can be clearly classified, though other axes data are not clearly clustered, e.g. Figure 6 . This suggests the up-down movement of left-knee may be essential for volleyball attack skill, and further investigation seems to be needed for certify this hypothesis.

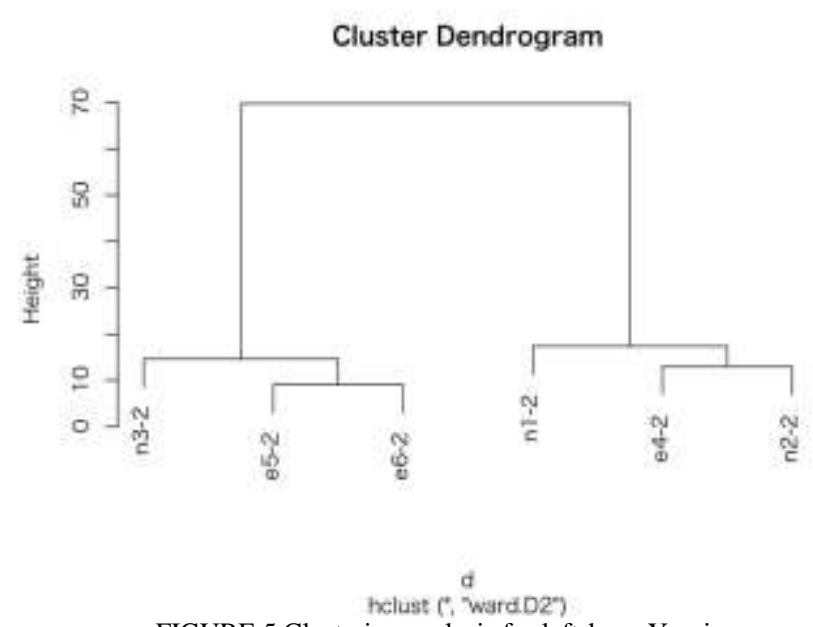

FIGURE 5.Clustering analysis for left-knee Y-axis.

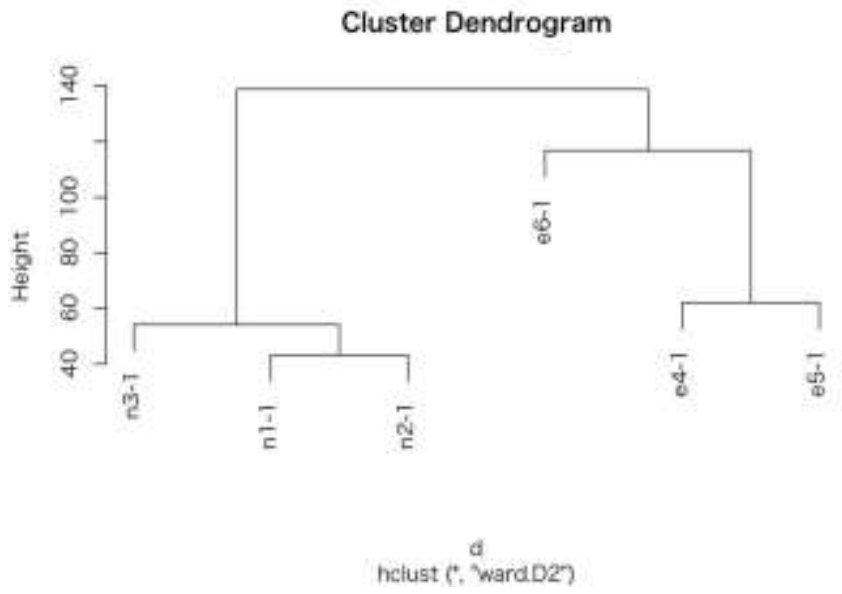

FIGURE 6. Clustering analysis for right-weist X-axis.

\section{CONCLUSION}

This paper presents sports skill classification using time series motion picture data, focused on volleyball. Volleyball play is analyzed with motion picture data recorded by hi-speed cam-coder. Time series data are obtained from the motion picture data with marking points, and analyzed using FFT and then clustering techniques. We classify technical skills of volleyball attacks.

As future plans, we have to progress further experiments, and measure more precise data and then analyze in details.

\section{ACKNOWLEDGMENT}

Part of this research was supported by JSPS KAKENHI Grant Number 15K02185, 15K03802 and 15K15818. This research was partially assisted, especially for data management, by Mr. Y. Tamari and Mr. Y. Tsujino at Hannan University. The authors greatly appreciate those. 


\section{REFERENCES}

[1] Y. Mochizuki, R. Himeno, and K. Omura, "Artificial skill and a new principle in sports (special issue on digital human : Measurement and modeling of human functions) (in japanese)," System, Control, and Information, vol. 46, no. 8, pp. 498-505, 2002.

[2] Y. Matsumoto, Organization and Skill - Organization Theory of Preservation of Technique (in Japanese). $\quad$ Hakuto Shobo, 2003.

[3] T. Maeda, M. Fujii, and I. Hayashi, "Time Series Data Analysis for Sport Skill," in Proceedings of the 2012 12th International Conference on Intelligent Systems Design and Applications (ISDA), Kochi (India), 2012, pp. 392-397.

[4] T. Maeda, M. Fujii, I. Hayashi, and T. Tasaka, "Sport Skill Classification Using Time Series Motion Picture Data," in Proceedings of the 40th Annual Conference of the IEEE Industrial Electronics Society (IECON 2014), Dallas (TX, USA), 2014, pp. 5272-5277.

[5] T. Maeda and M. Yajima, "Personal Skill Identification Using Time Series Motion Picture Data," in Proceedings of the 41th Annual Conference of the IEEE Industrial Electronics Society (IECON 2015), Yokohama (Japan), 2015, pp. 2475-2478.

[6] T. Nomura, S. Oshikawa, Y. Suzuki, K. Kiyono, and P. Morasso, "Modeling human postural sway using an intermittent control and hemodynamic perturbations," Mathematical Biosciences, vol. 245, iss.1, pp. 86-95, 2013.

[7] S. Wilkinson, "A training program for improving undergraduates' analytic skill in volleyball," Journal of Teaching in Physical Education, vol. 11, iss.2, pp. 177-194, 1992.

[8] K. Watanabe and M. Hokari, "Kinematical analysis and measurement of sports form," IEEE Transactions on Systems, Man and Cybernetics, Part A: Systems and Humans, vol. 36, iss.3, pp. 549-557, 2006.

[9] K. Barzouka, N. Bergeles, and D. Hatziharitos, "Effect of simultaneous model observation and self-modeling of volleyball skill acquisision," Perceptual and Motor Skills, vol. 104, pp. 32-42, 2007. 